\title{
An assessment of the haemodynamic alterations in acute myocardial infarction using the systolic time intervals
}

\author{
E. DAVID BENNETT, CHARLES S. SMITHEN, and \\ EDGAR SOWTON ${ }^{1}$
}

\author{
Institute of Cardiology and National Heart Hospital, London
}

\begin{abstract}
Systolic time intervals were measured in 24 patients with acute myocardial infarction and compared to normal values predicted for heart rate and sex. Pulmonary artery pressures were measured in 18 patients and cardiac outputs using the Fick technique in 10 patients. In all patients the QS2 interval (total electromechanical systole) was short during the acute episode. This shortening in the QS2 interval was due primarily to a reduction in left ventricular ejection time ( $\mathrm{r}=0.71 ; \mathrm{P}<0.001)$ with no change in the pre-ejection period. The mean deviation from normal of the QS2 interval was $27 \pm 3.6 \mathrm{msec}$ in patients with an uncomplicated clinical course and $52 \pm 4.7 \mathrm{msec}$ in patients with a complicated course, the difference being significant at the $1 \%$ level. Those patients with the highest pulmonary artery diastolic pressure tended to have the shortest QS2 intervals $(r=-0.58 ; \mathrm{P}<0.01)$. There was no relationship between stroke volume and the deviations in the QS2 and left ventricular ejection time intervals from normal. The implication of these findings is discussed.
\end{abstract}

The need of a bedside technique for measuring left ventricular function has been recognized by Weissler, Peeler, and Roehill, 1961 ; Weissler, Harris, and Schoenfeld, 1969). They have shown that the systolic time intervals in subjects with normal and abnormal hearts can be sensitive indicators of left ventricular performance (Weissler, Harris, and Schoenfeld, 1968 ; Garrard, Weissler, and Dodge, 1970).

Few studies of left ventricular function in patients with acute myocardial infarction have been carried out because of the risks inherent in catheterizing the left side of the heart. We have, therefore, measured the systolic time intervals in patients with acute myocardial infarction and related them to more standard haemodynamic measurements as an assessment of left ventricular function. Furthermore, we have evaluated their usefulness in the diagnosis and prognosis of this disease.

\section{METHODS}

The duration of the phases of left ventricular systole were measured from simultaneous recordings of the

tCorrespondence to: Edgar Sowton, Cardiac Department, Guy's Hospital, London S.E.1 electrocardiogram, phonocardiogram, and arterial ${ }^{3}$ pressure trace. The electrocardiographic lead most clearly demonstrating ventricular depolarization, usually lead 2, was used. A high-frequency phonocardiogram using a crystal contact microphone waso recorded from the left sternal border. The arterial trace was obtained from an indwelling Tefion catheteri inserted percutaneously into a femoral artery. A3. polyethylene catheter (PE.60) was introduced into anc antecubital vein and was flow guided into the pul- $\frac{3}{3}$ monary artery. Pressures were measured using a Statham P23Db electromanometer. The patients were 3 supine and the zero reference point was taken as $D$ mid-thorax. An Electronics for Medicine multi-ㅡㅡㄹ channel photographic recorder was used with a paper speed of $100 \mathrm{~mm} / \mathrm{sec}$. Cardiac outputs were measuredos using the Fick technique, expired gas being collected N in a plastic Douglas bag and the volume wasN measured in a Godart spirometer and converted to ${ }^{\omega}$ S.T.P. The gas was analysed on an Instrumentation? Laboratory blood gas analyser which had beeno frequently checked by gases of known concentrations $\widetilde{D}$ analysed by the micro-scholander technique. Arterial $\stackrel{?}{?}$ and mixed venous blood samples were withdrawn 0 simultaneously with the collection of expired gas ando analysed immediately on the Instrumentation Laboratory machine. The blood oxygen content was calcu- $\frac{P}{\mathbb{D}}$ lated from the oxygen tension using standard com-음 2Plysu Industrial Ltd., Woburn Sands, Bletchley 
puterized physiological tables (Kelman and Nunn, 1968). All measurements were made at a similar time in the morning with the patients fasting.

Figure 1 illustrates the manner in which the systolic intervals were obtained. The QS2 or total electromechanical interval was measured from the $Q$ wave of the electrocardiogram to the beginning of the first high-frequency component of the second heart sound on the phonocardiogram. Left ventricular ejection time was measured from the initial upstroke of the arterial pressure trace to the mid-point of the dicrotic notch. The pre-ejection period was calculated by subtracting the left ventricular ejection time from the QS2 interval. Only complexes which clearly demonstrated all the above features were used. Measurements were made to the nearest $5 \mathrm{msec}$. Five complexes were measured and the mean was calculated.

The systolic time intervals in the patients with acute myocardial infarction were compared with those obtained by Weissler in 211 normal subjects. Our measurements were made under similar conditions except that we measured LVET from a direct arterial trace rather than an external carotid trace. Other studies (Gibson and Sowton, 1970) in our laboratory with near normal subjects demonstrated a close correlation between ejection time measured by the present technique and the normal values published by Weissler. Bush et al. (1970) have shown that the LVET measured by the external carotid trace is within $3 \mathrm{msec}$ of the direct left ventricular ejection time. The deviations from the normal in the QS2 interval $(\triangle \mathrm{QS} 2)$ and LVET interval ( $\triangle$ LVET) were calculated as the difference between the observed interval and that predicted from the normal regression lines for heart rate and sex.

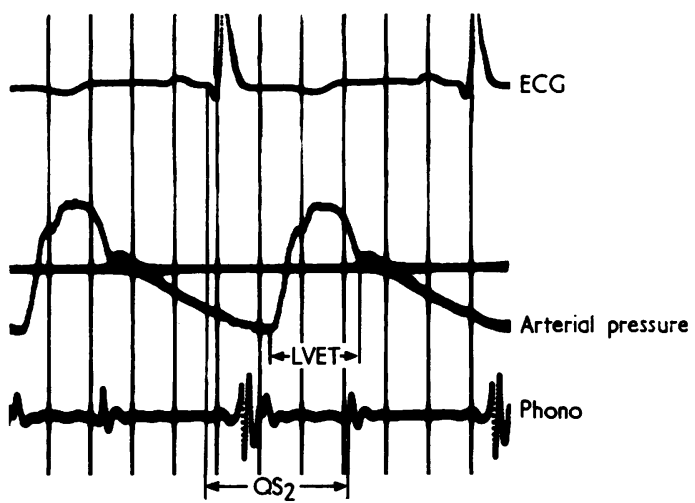

FIG. 1. Illustrates the method by which the systolic time intervals were measured from a typical recording. $L V E T=$ left ventricular ejection time; $Q S 2=$ total electromechanical systole. From above downwards: lead 2 of the electrocardiogram, arterial pressure tracing, phonocardiogram recorded at the left sternal edge. Paper speed $100 \mathrm{~mm} / \mathrm{sec}$. The time lines are 100 msec apart. Pre-ejection period was calculated by subtracting LVET from QS2.
MATERIAL

Twenty-seven patients with suspected acute myocardial infarction who were admitted to the coronary care unit of the National Heart Hospital were studied. Twenty-four of these patients subsequently exhibited criteria of myocardial infarction, namely, progressive ST segment and T-wave changes, the development of $Q$ waves and significant enzyme elevations.

Seventy-five daily measurements of the QS2 interval were made on the 24 patients with infarction. Nineteen measurements of LVET were made in 10 patients.

Thirty-five daily pulmonary artery pressure recordings were obtained on 18 patients and 17 cardiac output studies were performed on 10 patients. For technical reasons it was not possible to make all the measurements on every patient.

\section{RESULTS}

The pertinent clinical data and the results of all individual measurements are available from the authors upon request.

QS2 INTERVAL Figure 2 shows the QS2 intervals of patients with myocardial infarction plotted against heart rate and compared to the normal regression line obtained by Weissler. For graphic

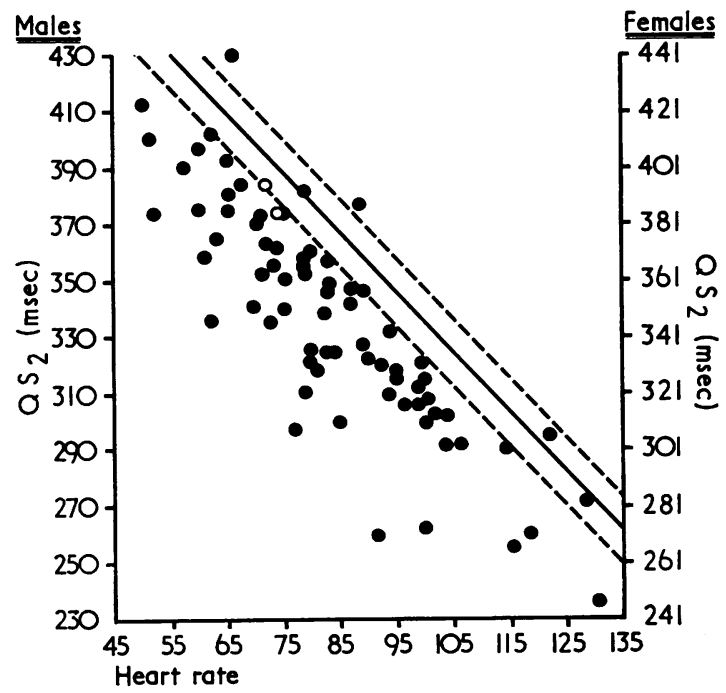

FIG. 2. Relationship of 75 measurements of QS2 to heart rate in 24 patients with acute myocardial infarction compared to the normal regression line of Weissler $(Q S 2=-2 \cdot 1 ; H . R .+546)$. The dotted lines represent one standard deviation. The vertical axes have been corrected for the mean difference of $11 \mathrm{msec}$ between males and females. The symbols $O$ represent measurements made at least 14 days after the acute episode. 
presentation the regression slope of the normal male group is shown and the vertical axes are corrected for the mean difference of $11 \mathrm{msec}$ between the normal males and females. In all patients the QS2 interval was short at some time during the acute episode and, of the 75 measurements, only six did not fall at least one standard deviation below the normal.

LVET Figure 3 shows the 19 measurements of left ventricular ejection time made in 10 patients plotted against heart rate. These data are again compared to the normal regression line with the correlation for sex. In 9 of the 10 patients the left ventricular ejection time was short during some stage of the acute illness, and 17 of the 19 measurements of left ventricular ejection time were more than one standard deviation below the normal.

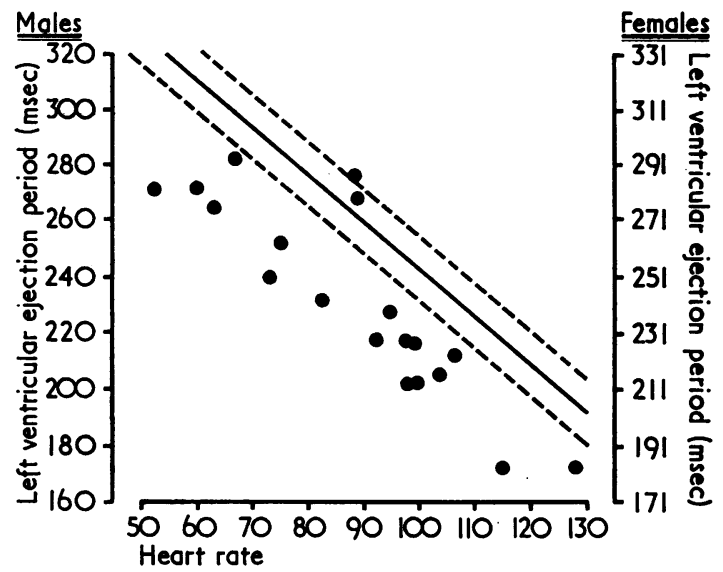

FIG. 3. Relationship of 19 measurements of LVET to heart rate in 10 patients with acute myocardial infarction compared to the normal regression line ( $L V E T=-1 \cdot 7$; H.R.+413). The dotted lines represent one standard deviation and the vertical axes have been correlated for the mean difference of $11 \mathrm{msec}$ between males and females.

PRE-EJECTION PERIOD The pre-ejection period in 10 patients is compared to the normal regression line in Fig. 4. As there is no sex difference for pre-ejection period no correction has been made. Of the 19 measurements, 15 were in the normal range, three just below normal, and one just above.

CLINICAL STATE AND QS2 The 24 patients were divided into two clinical groups. Group A consisted of eight patients with an entirely uncomplicated course. Group B consisted of 16 patients

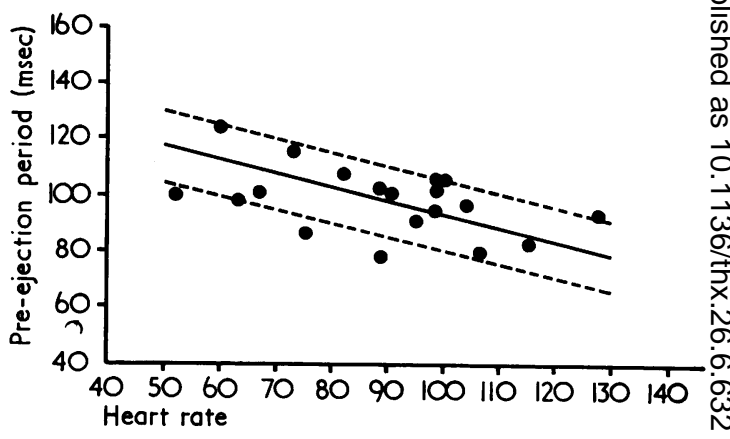

FIG. 4. Relationship of 19 measurements of pre-ejection period to heart rate in 10 patients with acute myocardial infarction compared to the normal regression line $(P E P=O$ $-0.4 ; H . R .+131)$. The dotted lines represent one standard deviation.

with a complicated clinical course, namely, signifi- $\vec{\theta}$ cant dysrhythmias and/or left ventricular failure.Figure 5 shows the QS2 deviation for both groups. The mean in group $A$ was $-27 \pm 3.6 \mathrm{msec}$ and in group B $-52 \pm 4.7 \mathrm{msec}$. There was a statistically significant difference between these mean values $(\mathbf{P}<0.01)$.

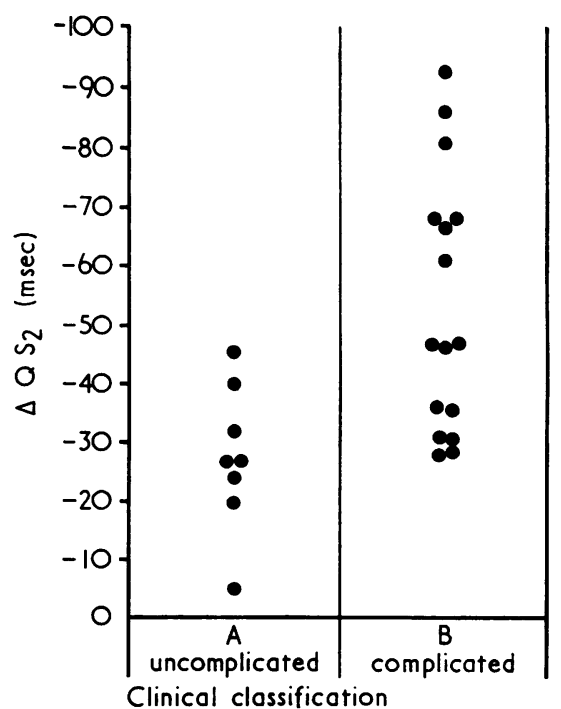

FIG. 5. Relationship of the deviation in QS2 from the normal $(\triangle Q S 2)$ and the clinical state in 24 patients with음 acute myocardial infarction. $A=$ uncomplicated; $B=$ significant dysrhythmias and/or left ventricular failure. The mean $Q S 2$ deviation of group $A=-27 \pm 3.6$ msec. The mean $Q S 2$ deviation of group $B=-52 \pm 4.7$ msec ( $P<0.01)$. 
PULMONARY ARTERY PRESSURE AND QS2 The pulmonary artery diastolic pressure was used as an indicator of left ventricular end diastolic pressure (Kaltman, Herbert, Conroy, and Kossman, 1966 ; Abbott, 1970 ; Jenkins, Bradley, and Branthwaite, 1970). Figure 6 demonstrates the relationship between the deviation in QS2 interval $(\triangle \mathrm{QS} 2)$ and the pulmonary artery diastolic pressure: $\mathrm{QS2}=$ 24.1-1.44 pulmonary artery diastolic pressure. There was a moderate correlation between these two variables $(r=0.58 ; P<0.01)$.

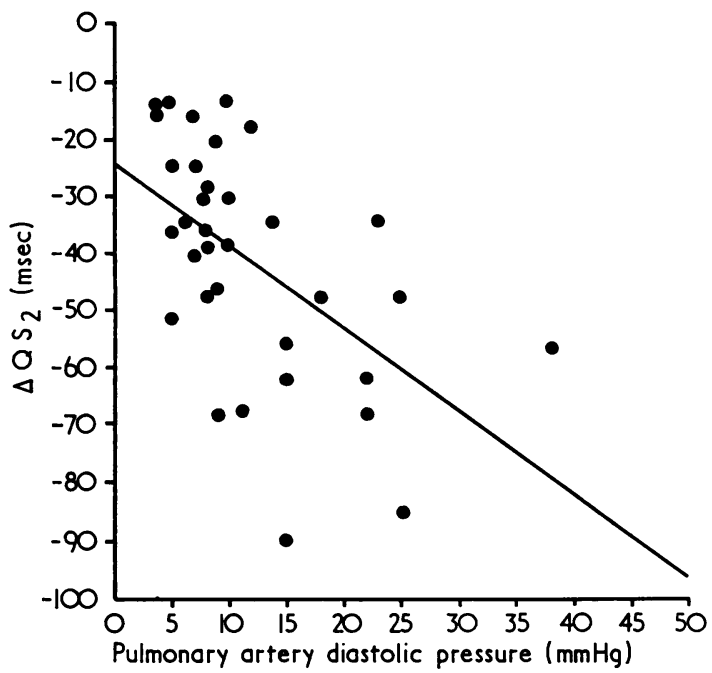

FIG. 6. Relationship between the deviation in QS2 interval ( $\triangle Q S 2)$ and the pulmonary artery diastolic pressure; $Q S 2=-24.4-1.44$ pulmonary artery diastolic pressure $(r=-0.58 ; P<0.01)$.

$\triangle \mathrm{QS} 2$ AND $\triangle$ LVET Figure 7 shows the relationship between the deviations in QS2 and LVET lying along the line of identity: $Q S 2=-16.0+0.6$ LVET $(r=0.71 ; P<0.001)$.

$\triangle$ QS2, $\triangle$ LVET, AND STROKE VOLUME Figure 8 demonstrates that there was no significant relationship between the stroke volume and the deviations in QS2 and left ventricular ejection time.

\section{DISCUSSION}

The validity of the measurements of systolic time intervals in both the normal and abnormal heart has been well substantiated (Weissler et al., 1961 ; Weissler et al., 1968 and 1969 ; Garrard et al., 1970). However, little information is available on these intervals in patients with acute myocardial

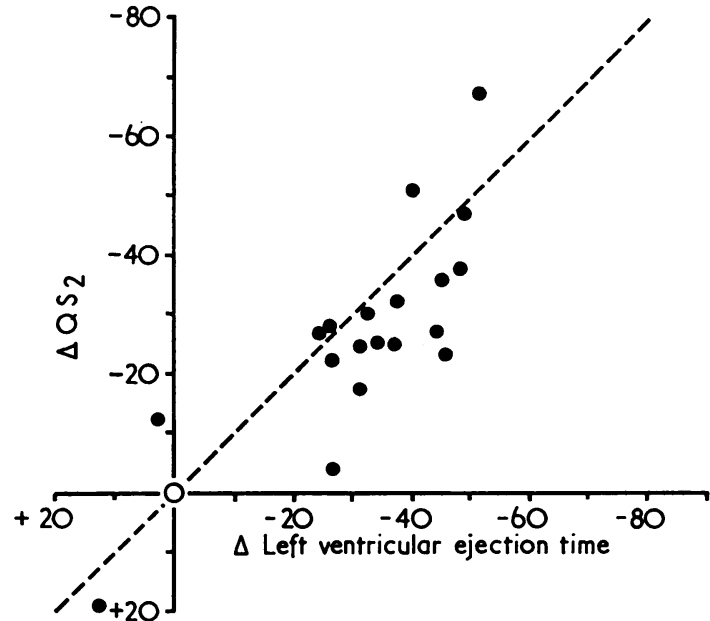

FIG. 7. Relationship between the deviations in $Q S 2$ $(\triangle Q S 2)$ and left ventricular ejection time ( $\triangle L V E T)$; $Q S 2=-16.0+0.6$ LVET $(r=0.71 ; P<0.001)$. The dotted line represents the line of identity.

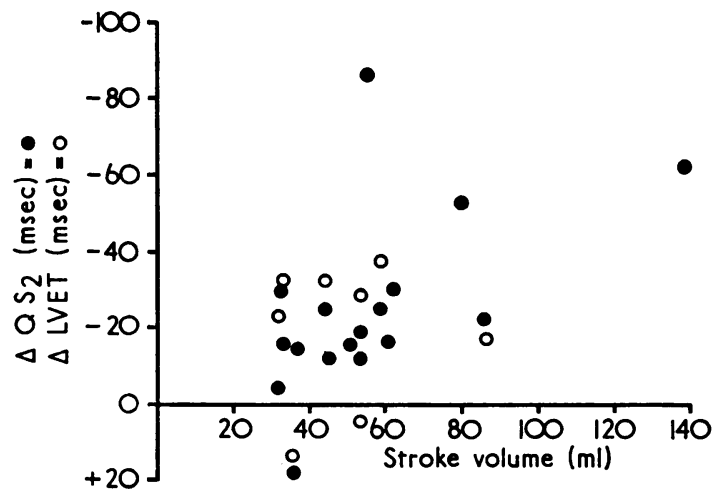

FIG. 8. Relationship between stroke volume and the deviations in QS2 and LVET.

infarction. The present study demonstrates that these patients have short QS2 intervals, which is in agreement with Toutouzas, Gupta, Samson, and Shillingford (1969), who found similar shortenings in 20 patients, and with Diamant and Killip (1970), who found reductions in 100 patients. We have further demonstrated that the LVET is reduced in acute myocardial infarction but in our small series we were unable to show any change in the preejection period. Gunnar et al. (1970) demonstrated similar reductions in LVET and were also unable to find any change in pre-ejection period. Diamant has also found reductions in LVET but demonstrated a cocomitant increase in the pre-ejection period. 
The significant relationship between clinical state and the degree of shortening of the QS2 interval suggests that the more severe the myocardial infarction, the greater the reduction in this interval. This is further substantiated by the observation that those patients with the highest pulmonary artery diastolic pressures tended to have the shortest QS2 intervals. These findings suggest that measurement of the QS2 interval may be helpful in the diagnosis and prognosis of acute myocardial infarction and its subsequent course.

The cause of the short QS2 interval in acute myocardial infarction is uncertain but we have demonstrated that the change which occurs is most probably due to a reduction in left ventricular ejection time, as is demonstrated in Fig. 7. Factors which are known to determine the left ventricular ejection time include heart rate, stroke volume, aortic pressure, and the contractile state of the myocardium (Braunwald, Sarnoff, and Stainsby, 1958). The latter may be influenced by agents such as catecholamines (Wallace, Mitchell, Skinner, and Sarnoff, 1963), digitalis (Braunwald et al., 1958), and calcium. Figure 2 shows that despite widely varying heart rates there was a consistent deviation of the QS2 interval from the normal. Our patients did not have a significant change in mean aortic pressure, so that neither of these factors appears to account for our findings. Although it has been postulated that the short left ventricular ejection time in acute myocardial infarction is due to a reduction in stroke volume (Diamant and Killip, 1970) we were unable to find any relationship between stroke volume and the deviations in QS2 and LVET from normal in our series. This may be explained by possible alterations in end-diastolic volume. Although enddiastolic volume was not measured, our findings of a correlation between pulmonary artery diastolic pressure and QS2 shortening suggest that an increase in end-diastolic volume was associated with a reduction in the QS2 interval, reflecting a reduction in LVET. Garrard et al. (1970) have demonstrated a good correlation between ejection fraction measured angiographically and the duration of left ventricular ejection. It seems probable that an alteration in the contractile state of the myocardium leading to a decrease in ejection fraction after acute myocardial infarction could be an important factor accounting for our findings.

Another factor to be considered is the level of circulating catecholamines after myocardial infarction. Braunwald et al. (1958) has shown that in the isolated heart-lung preparation infusion of sympathomimetic amines, maintaining all other para- meters constant, resulted in a significant reduction of the left ventricular ejection time. Wallace $e t$ al. (1963) infused noradrenaline into six dogs and found both the total electromechanical interval $\triangle$ and the left ventricular ejection time shortened. के Since a number of investigators have shown a $\overrightarrow{0}$ significant increase in urinary catecholamine levels after acute myocardial infarction (Valori, Thomas, $\vec{\omega}$ and Shillingford, 1967 ; Wallace, 1968), this increased level of sympathetic activity may be $\times$ influencing the systolic time intervals. N

In conclusion we have found the measurement of systolic time intervals valuable in assessing alterations in left ventricular function after acute myocardial infarction and helpful as diagnostic and prognostic indicators. However, further study is required for a clearer understanding of the mechanisms responsible for the shortened left ventricular ejection time and QS2 intervals in this condition.

\section{REFERENCES}

Abbott, J. A. (1970). Pulmonary artery diastolic pressure reflecting change in left heart filling pressure. Cardiovasc. Res., VI World Congress of Cardiology, Abstracts of papers, 58.

Braunwald, E., Sarnoff, E. J., and Stainsby, W. N. (1958). Determinants of duration and mean rate of ventricular ejection. Circulat. Res., 6, 319.

Bush, C. A., Lewis, R. P., Leighton, R. F., Fontana, M. E., and Weissler, A. M. (1970). Verification of systolic time intervals and the true isovolumic contraction time from the apexcardiogram by micromanometer catheterization of the left ventricle and aorta. (Abstr.) Circulation, 42, (Suppl. III), III-121.

Diamant, B., and Killip, T. (1970). Indirect assessment of 으 left ventricular performance in acute myocardial infarction. Circulation, 42, 579.

Garrard, C. L. Jr., Weissler, A. M., and Dodge, H. T. (1970). The relationship of alterations in systolic time intervals to ejection fraction in patients with cardiac disease. Circulation, 42, 455.

Gibson, D., and Sowton, G. E. (1970). Personal commu- 음 nication.

Gunnar, R., Sinno, Z., Loeb, H., Bessinger, H., Chuquimia, R., and Rahimtoola, S. H. (1970). Left ventricular performance during first two days after acute myo- N cardial infarction. (Abstr.) Circulation, 42, (Suppl. III), III-162.

Jenkins, B. S., Bradley, R. D., and Branthwaite, M. A. N (1970). Evaluation of pulmonary arterial end-diastolic $\omega$ pressure as an indirect estimate of left atrial mean pressure. Circulation, 42, 75.

Kaltman, A. J., Herbert, W. H., Conroy, R. J., and Kossman, C. E. (1966). The gradient in pressure across the pul- क्S monary vascular bed during diastole. Circulation, 34, 377.

Kelman, G. R., and Nunn, J. F. (1968). Computer Produced Physiological Tables. Butterworth, London.

Toutouzas, P., Gupta, D., Samson, R., and Shillingford, J. @ (1969). Q-second sound interval in acute myocardial infarction. Brit. Heart J., 31, 462. 
Valori, C., Thomas, M., and Shillingford, J. (1967). Free noradrenaline and adrenaline excretion in relation to clinical syndromes following myocardial infarction. Amer. J. Cardiol., 20, 605.

Wallace, A. G. (1968). Catecholamine metabolism in patients with acute myocardial infarction. In Acute Myocardial Infarction, Proc. Symposium, Edinburgh 1967, Edited by D. G. Julian, and M. F. Oliver, p. 237. Livingstone, Edinburgh.

Mitchell, J. H., Skinner, A. S., and Sarnoff, S. J. (1963). Duration of the phases of left ventricular systole. Circulat. Res., 12, 611.
Weissler, A. M., Harris, W. S., and Schoenfeld, C. D. (1968). Systolic time intervals in heart failure in man. Circulation, 37, 149.

(1969). Bedside technics for the evaluation of ventricular functions in man. Amer. J. Cardiol., 23, 577.

Peeler, R. G., and Roehill, W. H. (1961). Relationships between left ventricular ejection time, stroke volume, and heart rate in normal individuals and patients with cardiovascular disease. Amer. Heart J., 62, 367. 